\title{
Inventaire printanier d'une frayère multispécifique : l'ichtyofaune des rapides de la rivière Gatineau, Québec
}

\author{
Annie Comtois $^{1}$, François Chapleau ${ }^{1}$, Claude B. Renaud ${ }^{2}$, Henri Fournier ${ }^{3}$, Brent Campbell $^{1}$, \\ et RICHARD PARISEAU ${ }^{3}$
}

${ }^{1}$ Département de biologie, Université d'Ottawa, Ottawa, Ontario K1N 6N5 Canada

${ }^{2}$ Division de la recherche, Musée canadien de la nature, Casier Postal 3443, Succursale D, Ottawa, Ontario K1P 6P4 Canada

${ }^{3}$ Direction de l'aménagement de la faune de l'Outaouais, Société de la faune et des parcs du Québec, Gatineau, Québec J8Y 3R7 Canada

Comtois, Annie, François Chapleau, Claude B. Renaud, Henri Fournier, Brent Campbell, et Richard Pariseau. 2004. Inventaire printanier d'une frayère multispécifique : l'ichtyofaune des rapides de la rivière Gatineau, Québec. Canadian FieldNaturalist 118(4): 521-529.

Un inventaire ichtyologique printanier a été réalisé dans la rivière Gatineau, dans le premier rapide à l'amont de son embouchure dans la rivière des Outaouais, pour établir la chronoséquence d'utilisation du site par les espèces s'y reproduisant, dont trois espèces de chevaliers. L'échantillonnage des rapides a permis de capturer 2388 poissons répartis en 13 familles et 39 espèces, dont six ayant fait l'objet d'une évaluation par le COSEPAC. Seize espèces étaient en état de se reproduire lors de l'échantillonnage. Nous avons observé une reproduction plus ou moins simultanée pour le Chevalier blanc et le Chevalier rouge, suivie de celle du Chevalier de rivière. Également, pour ces trois espèces, nous avons observé la capture des mâles de stade V plus tôt que les femelles, et nous les retrouvons en plus grand nombre. Ceci pourrait s'expliquer du fait que les mâles précèdent les femelles sur les frayères, que celles-ci sont reconnues pour rester très peu de temps sur les sites de fraie et qu'un minimum de deux mâles par femelle est requis pour la reproduction. Cette étude démontre que les rapides de la rivière Gatineau doivent être protégés puisqu'ils sont habités par trois espèces qui ont obtenu un statut spécial du COSEPAC (Menacée: Fouille-roche gris; situation préoccupante : Lamproie du nord et Chevalier de rivière) en plus de constituer une frayère multispécifique importante.

Mots-clés: Rapides, Rivière Gatineau, Outaouais, Fraie, Chevalier blanc, Moxostoma anisurum, Chevalier de rivière, Moxostoma carinatum, Chevalier rouge, Moxostoma macrolepidotum, Lamproie du nord, Ichthyomyzon fossor, Fouille-roche gris, Percina copelandi.

A spring survey of fishes was conducted in the Gatineau River (Quebec), in the first rapids upstream of its confluence with the Ottawa River, to establish the fish spawning chronosequence, especially for the three species of redhorses. The sampling resulted in the capture of 2388 fish belonging to 13 families and 39 species. Six of these species have been assessed by COSEWIC. Sixteen species were in spawning readiness. Spawning of the Silver Redhorse and Shorthead Redhorse occurred first, followed by the River Redhorse. Stage V males of the three redhorse species were captured earlier and in greater numbers than females. These observations are consistent with other studies which suggest that males precede females to the spawning grounds, that females remain there for a short period and that a minimum two males per female is required for reproduction. This study shows that the Gatineau River rapids must be protected because they harbor three species that have obtained a status by COSEWIC (Threatened: Channel Darter; Special concern: Northern Brook Lamprey and River Redhorse) as well as being an important multi-species spawning ground.

Key Words: Rapids, Gatineau River, Outaouais, spawning, Silver Redhorse, Moxostoma anisurum, River Redhorse, Moxostoma carinatum, Shorthead Redhorse, Moxostoma macrolepidotum, Northern Brook Lamprey, Ichthyomyzon fossor, Channel Darter, Percina copelandi.

Un inventaire réalisé au printemps 1989 à l'aide de filets de dérive a permis de constater que les rapides de la rivière Gatineau en aval du barrage hydroélectrique de Farmer's Point (Gatineau, Québec) (Figure 1) sont utilisés pour la reproduction de l'Esturgeon jaune, des Percidae (Sander spp. et Perca flavescens), et des Catostomidae (Catostomus spp. et Moxostoma spp.) (Houde et Fournier 1992). Une pêche expérimentale réalisée en 1998 (données non publiées) a révélé la présence à ce site d'une concentration exceptionnelle de Chevalier de rivière. Des travaux furent donc entrepris en 1999 pour décrire cette population et son utilisation du rapide Farmer's.
L'étude ichtyologique de ce secteur de la rivière revêt un intérêt particulier parce que le barrage agit comme barrière au déplacement des poissons vers l'amont. Ceci pourrait occasionner une concentration de poissons dans le secteur des rapides en aval du barrage, en particulier au printemps, lors des migrations reproductrices. Ainsi, le secteur en aval du barrage pourrait être un site privilégié pour la reproduction des poissons provenant des rivières Gatineau et des Outaouais.

Le but de notre étude est de présenter l'inventaire printanier des espèces de poissons de ce secteur de la rivière alors que plusieurs de celles-ci sont normalement en période de fraie. De plus, une attention particu- 




Figure 1. Site de la rivière Gatineau près du pont Alonzo-Wright, se trouvant à $5,2 \mathrm{~km}$ de la jonction avec la rivière des Outaouais.

lière sera portée sur la répartition dans le temps de la reproduction des trois espèces de chevalier (Moxostoma spp.) qui utilisent le secteur des rapides pour frayer.

\section{Site d'échantillonnage}

La section de la rivière Gatineau $\left(45^{\circ} 29^{\prime} \mathrm{N}\right.$, $\left.75^{\circ} 44^{\prime} \mathrm{O}\right)$ échantillonnée se trouve entre 6,0 et $5,2 \mathrm{~km}$ de l'embouchure sur la rivière des Outaouais (Figure 1). La zone d'échantillonnage couvre une longueur de $800 \mathrm{~m}$ et est délimitée en amont par le pont AlonzoWright et à l'aval par l'extrémité d'une île localisée près de la rive ouest de la rivière. La largeur de la rivière dans ce secteur varie de $170 \mathrm{~m}$ en amont à $330 \mathrm{~m}$ en aval et couvre une superficie d'environ 22 ha. La profondeur de l'eau varie de 0,5 à $5 \mathrm{~m}$. Le courant est particulièrement rapide sur une distance d'environ $400 \mathrm{~m}$ à l'aval du pont et la vitesse devient beaucoup plus lente par la suite alors que la rivière s'élargit et devient plus profonde.

Dans les eaux rapides, le substrat est composé de galets, de cailloux et de gros blocs épars dans le centre de la rivière et de gravier en rive ouest. La rive est a été largement modifiée par des remblayages et la construction de quais et murs de soutènement. En aval, où la rivière est plus large et le courant plus lent, le fond est constitué de gravier, de cailloux et de galets; en rive ouest, le substrat est composé de sable 
TABLEAU 1. Espèces recensées lors de l'échantillonnage du rapide Farmer's, rivière Gatineau, au printemps 1999.

\begin{tabular}{|c|c|c|c|}
\hline Famille & Nom latin (Espèce) & Nom commun français & $\begin{array}{c}\text { Nombre } \\
\text { d'individus }\end{array}$ \\
\hline \multirow[t]{3}{*}{ Petromyzontidae } & Ichthyomyzon unicuspis & Lamproie argentée & 8 \\
\hline & Ichthyomyzon fossor & Lamproie du nord & 1 \\
\hline & Lampetra appendix & Lamproie de l'est & 31 \\
\hline Acipenseridae & Acipenser fulvescens & Esturgeon jaune & 19 \\
\hline Hiodontidae & Hiodon tergisus & Laquaiche argentée & 276 \\
\hline Anguillidae & Anguilla rostrata & Anguille d'Amérique & 1 \\
\hline \multirow[t]{6}{*}{ Cyprinidae } & Luxilus cornutus & Méné à nageoires rouges & 4 \\
\hline & Notemigonus crysoleucas & Méné jaune & 2 \\
\hline & Notropis atherinoides & Méné émeraude & 309 \\
\hline & Notropis hudsonius & Queue à tache noire & 2 \\
\hline & Rhinichthys cataractae & Naseux des rapides & 1 \\
\hline & Semotilus corporalis & Ouitouche & 52 \\
\hline \multirow[t]{5}{*}{ Catostomidae } & Carpiodes cyprinus & Couette & 68 \\
\hline & Catostomus commersoni & Meunier noir & 33 \\
\hline & Moxostoma anisurum & Chevalier blanc & 223 \\
\hline & Moxostoma carinatum & Chevalier de rivière & 270 \\
\hline & Moxostoma macrolepidotum & Chevalier rouge & 254 \\
\hline \multirow[t]{3}{*}{ Ictaluridae } & Ameiurus nebulosus & Barbotte brune & 9 \\
\hline & Ictalurus punctatus & Barbue de rivière & 57 \\
\hline & Noturus insignis & Chat-fou liséré & 3 \\
\hline \multirow[t]{2}{*}{ Esocidae } & Esox lucius & Grand brochet & 1 \\
\hline & Esox masquinongy & Maskinongé & 4 \\
\hline \multirow{2}{*}{ Salmonidae } & Salmo trutta & Truite brune & 1 \\
\hline & Salvelinus namaycush & Touladi & 1 \\
\hline Percopsidae & Percopsis omiscomaycus & Omisco & 5 \\
\hline \multirow[t]{6}{*}{ Centrarchidae } & Ambloplites rupestris & Crapet de roche & 116 \\
\hline & Lepomis gibbosus & Crapet-soleil & 9 \\
\hline & Lepomis macrochirus & Crapet arlequin & 4 \\
\hline & Micropterus dolomieu & Achigan à petite bouche & 17 \\
\hline & Micropterus salmoides & Achigan à grande bouche & 1 \\
\hline & Pomoxis nigromaculatus & Marigane noire & 22 \\
\hline \multirow[t]{7}{*}{ Percidae } & Etheostoma nigrum & Raseux-de-terre noir & 1 \\
\hline & Etheostoma olmstedi & Raseux-de-terre gris & 12 \\
\hline & Perca flavescens & Perchaude & 79 \\
\hline & Percina caprodes & Fouille-roche zébré & 315 \\
\hline & Percina copelandi & Fouille-roche gris & 76 \\
\hline & Sander canadensis & Doré noir & 16 \\
\hline & Sander vitreus & Doré jaune & 80 \\
\hline Sciaenidae & Aplodinotus grunniens & Malachigan & 5 \\
\hline
\end{tabular}

et de limon alors que la rive est a aussi été largement modifiée par les activités humaines.

\section{Méthodes}

L'échantillonnage s'est effectué à intervalle de 1 à 5 jours, du 29 avril au 23 juin 1999, entre 19:00 h et 01:00 h. Il s'est fait à l'aide d'un bateau de pêche électrique de marque Smith Root 16R. Des voltages de 250 à $1000 \mathrm{~V}$ et des ampérages de 0,25 à 3,00 A ont été utilisés selon la conductivité de l'eau. La pêche débutait à $20 \mathrm{~m}$ des piliers du pont Alonzo-Wright. Une dérive contrôlée du bateau était faite sur des transects de 400 à $800 \mathrm{~m}$ le long des rives et au centre de la rivière. La période de pêche active (lorsque le courant était émis dans l'eau) variait de 320 à 1732 s. Les poissons capturés étaient déposés dans un vivier installé sur le pont du bateau. Une fois le vivier rempli, la pêche active était arrêtée et les poissons étaient examinés et relâchés, sauf pour quelques spécimens qui ont été conservés pour des études ultérieures.

Les poissons étaient identifiés, dénombrés et le sexe ainsi que le stade de reproduction étaient déterminés lorsque cela était possible. Pour les chevaliers, le sexe était établi à partir de la présence de tubercules nuptiaux (Scott et Crossman 1974). Le stade de reproduction était déterminé par des caractéristiques morphologiques externes et selon l'index de maturité des gonades de Nikolsky (1963). Celui-ci classe les stades en six catégories. Les stades I, II et III, ne sont pas identifiables puisqu'ils reflètent des états de maturation des gonades qui ne montrent pas de signes externes. Le stade IV est caractérisé par l'identification de gonades fermes et gonflées au toucher de la paroi abdominale sans expulsion d'œufs ou de sperme. Le stade $\mathrm{V}$ est identifié lorsqu'il y a expulsion de produits sexuels par simple pression de l'abdomen. 


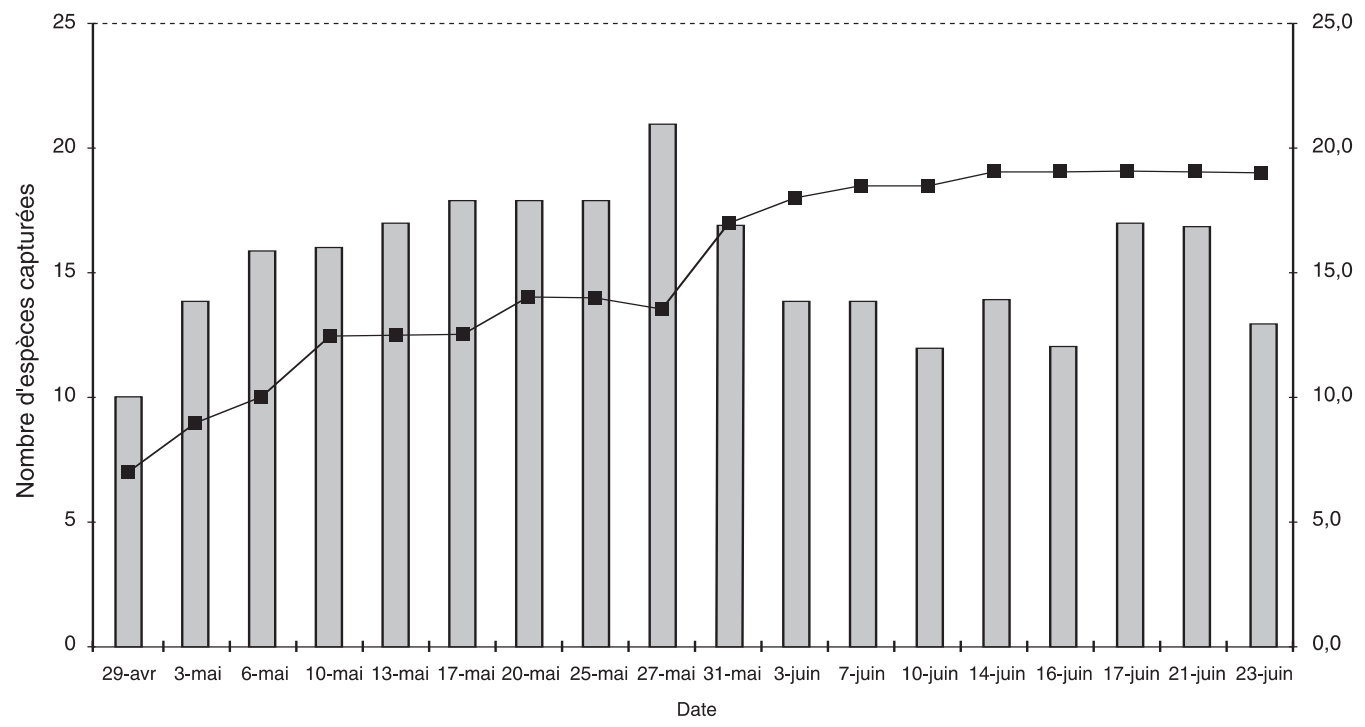

FIGURE 2. Évolution temporelle du nombre d'espèces capturées et de la température de l'eau (a) au rapide Farmer's, printemps 1999.

Un poisson au stade VI a un abdomen relativement flasque et sans expulsion massive de produits sexuels par pression abdominale.

L'échantillonnage ciblant surtout les chevaliers, l'abondance des espèces de petite taille est probablement une sous-estimation de leur présence dans le milieu.

\section{Résultats}

Familles et espèces capturées

L'échantillonnage des rapides de la rivière Gatineau a permis de capturer 2388 poissons en 12 heures de pêche active réparties sur 18 jours entre le 29 avril et le 23 juin 1999. Les captures se répartissent en 13 familles et 39 espèces (Tableau 1). Les Percidae dominent pour le nombre d'espèces capturées (sept espèces). Les Cyprinidae et les Centrarchidae sont représentés par six espèces chacune. Les Catostomidae dominent pour le nombre de spécimens (848), les chevaliers constituant $88 \%$ des captures. Un seul spécimen des Anguillidae (Anguilla rostrata) a été capturé (Tableau 1). D'ailleurs, un seul spécimen a été capturé pour huit espèces (Tableau 1) alors que plus de 300 spécimens ont été capturés pour le Méné émeraude (309) et le Fouille-roche zébré (315).

Six des espèces capturées ont fait l'objet d'une évaluation par le Comité sur la situation des espèces en péril au Canada (COSEPAC 2003) : l'Esturgeon jaune (non en péril; 1986), le Chevalier de rivière (situation préoccupante; 1987), le Chat-fou liséré (menacé; 1989 et données insuffisantes lors de la révision de 2002), la Lamproie du nord (situation préoccupante; 1991), le Fouille-roche gris (menacé; 1993, 2002) et le Raseux-de-terre gris (non en péril; 1993). Deux de ces espèces, le Chevalier de rivière et le Fouille-roche gris ont été capturés en grand nombre dans la rivière Gatineau, soit 270 et 76 individus respectivement (Tableau 1).

Comme nous pouvons le constater à la Figure 2, le plus petit nombre d'espèces capturées a été de dix lors de la première sortie, le 29 avril. Il a augmenté graduellement pour atteindre un maximum de 21 espèces le 27 mai. Par la suite, le nombre a diminué, variant de 12 à 17 espèces par sortie.

\section{Reproduction}

Des individus de 16 des espèces capturées étaient au stade $\mathrm{V}$ du cycle de reproduction et semblent donc y avoir frayé (Tableau 2). Pour plusieurs de ces espèces, nos captures permettent de délimiter une période de reproduction probable et les températures auxquelles elle se serait produite. Pour quelques espèces, des individus en état de fraie (stade V) étaient présents dès la première journée d'échantillonnage ou encore présents la dernière journée. Dans ces cas, il est possible que la période de reproduction s'étende au-delà de la période d'échantillonnage. Des individus des deux sexes de tous les stades avancés de développement des gonades (stades IV, V, VI) ont été observés pour cinq espèces seulement : le Chevalier blanc (Moxostoma anisurum), le Chevalier de rivière (M. carinatum), le Chevalier rouge (M. macrolepidotum), la Couette (Carpiodes cyprinus) et la Laquaiche argentée (Hiodon tergisus).

\section{Reproduction des trois espèces de chevalier}

L'abondance des captures permet d'établir la chronoséquence des périodes de reproduction des trois espèces de chevaliers dans la rivière Gatineau (Figures 3 et 4). Pour toutes les espèces, des spécimens de 
TABleau 2. Période de capture d'individus en fraie (stade V), selon le sexe et l'espèce, au rapide Farmer's, printemps 1999.

\begin{tabular}{|c|c|c|c|c|}
\hline \multirow[t]{2}{*}{ Espèce } & \multicolumn{2}{|c|}{ Nombre d'individus } & \multirow[t]{2}{*}{ Période de capture } & \multirow{2}{*}{$\begin{array}{c}\text { Température de } \\
\text { l'eau }\left({ }^{\circ} \mathrm{C}\right)^{1}\end{array}$} \\
\hline & $\sigma^{7}$ & Q & & \\
\hline Ambloplites rupestris & 2 & 3 & 27 mai - 10 juin & $13,5-18,5$ \\
\hline Aplodinotus grunniens & 1 & 1 & $3-6$ mai & $9,0-10,0$ \\
\hline Carpiodes cyprinus & 35 & 7 & 29 avril -3 juin & $7,0-18,0$ \\
\hline Catostomus commersoni & 8 & 5 & 29 avril - 27 mai & $7,0-13,5$ \\
\hline Hiodon tergisus & 198 & 2 & 10 mai - 23 juin & $12,5-19,0$ \\
\hline Ichthyomyzon fossor & 0 & 1 & 23 juin & 19,0 \\
\hline Ichthyomyzon unicuspis & 2 & 1 & 13 mai - 23 juin & $12,5-19,0$ \\
\hline Lampetra appendix & 5 & 3 & $3-20$ mai & $9,0-14,0$ \\
\hline Moxostoma anisurum & 89 & 6 & $3-31$ mai & $9,0-17,0$ \\
\hline Moxostoma carinatum & 96 & 16 & 10 mai - 23 juin & $12,5-19,0$ \\
\hline Moxostoma macrolepidotum & 67 & 7 & 29 avril -21 juin & $7,0-19,0$ \\
\hline Percina caprodes & 10 & 0 & $6-25$ mai & $10,0-14,0$ \\
\hline Percina copelandi & 7 & 1 & 20 mai - 21 juin & $14,0-19,0$ \\
\hline Percopsis omiscomaycus & 0 & 2 & 6 mai & 10,0 \\
\hline Pomoxis nigromaculatus & 3 & 3 & $3-13$ mai & $9,0-12,5$ \\
\hline Semotilus corporalis & 1 & 1 & $3-20$ mai & $9,0-14,0$ \\
\hline
\end{tabular}

${ }^{1}$ Température de l'eau au début et à la fin de la période de capture.

stade de maturité IV étaient présents au début de la période d'échantillonnage, le 29 avril, sauf pour les femelles du Chevalier rouge et les mâles du Chevalier de rivière. Pour ceux-ci, les individus au stade IV sont apparus le 3 et le 6 mai respectivement (Figure 3). Des mâles de stade IV ont été capturés jusqu'au
17 mai dans le cas du Chevalier rouge, jusqu'au 27 mai pour le Chevalier blanc et jusqu' au 7 juin pour le Chevalier de rivière. Les dernières femelles de stade IV de Chevaliers rouge et blanc ont été capturées le 17 mai, alors qu'elles ont été présentes sur le site jusqu'au 17 juin pour le Chevalier de rivière.

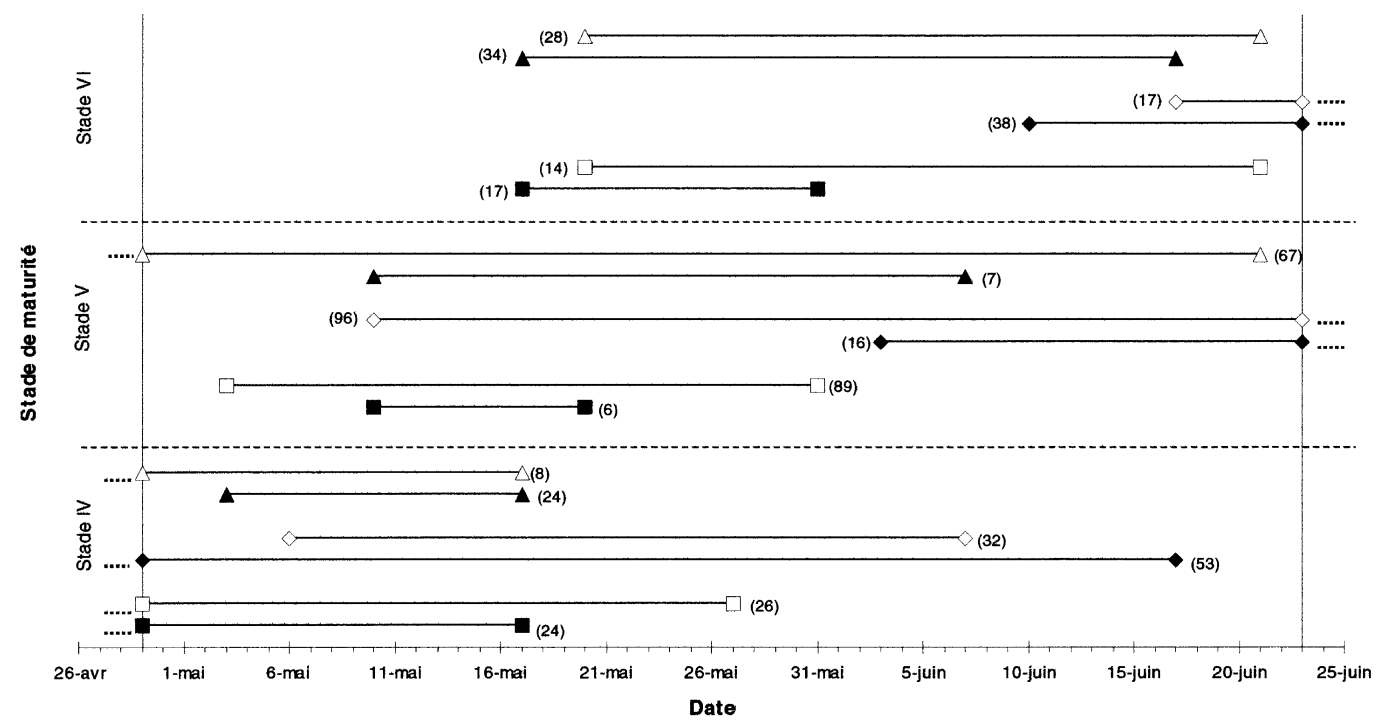

FIguRE 3. Période de reproduction des trois espèces de chevalier selon l'index de maturité de Nikolsky (1963) dans la rivière Gatineau, au printemps 1999. Le carré représente le Chevalier blanc $(\boldsymbol{\square})$, le losange, le Chevalier de rivière $(\bullet)$ et le triangle, le Chevalier rouge ( $\mathbf{\Delta})$. Les symboles pleins désignent les femelles tandis que les vides représentent les mâles. Le nombre d'individus capturés est indiqué entre parenthèses. Les lignes verticales correspondent au début et à la fin de la période d'échantillonnage. Le pointillé à l'extérieur de la période d'échantillonnage signifie que des individus appartenant aux stades indiqués ont pu se retrouver sur la frayère avant ou après cette période. 
Des mâles au stade V sont apparus dans les captures le 3 mai pour le Chevalier blanc et le 10 mai pour le Chevalier de rivière, à une température de 9,0 et $12,5^{\circ} \mathrm{C}$ respectivement. Les mâles Chevalier rouge de ce stade de maturité étaient présents dès le début de l'échantillonnage. Les femelles de stade $\mathrm{V}$ sont apparues le 10 mai pour le Chevalier blanc et le Chevalier rouge $\left(12,5^{\circ} \mathrm{C}\right)$ et le 3 juin pour le Chevalier de rivière $\left(18,0^{\circ} \mathrm{C}\right)$. Les captures de mâles au stade $\mathrm{V}$ sont toujours nettement plus abondantes que celles des femelles. Aucune femelle de stade V n'a été capturée après le 20 mai dans le cas du Chevalier blanc $\left(14,0^{\circ} \mathrm{C}\right)$ et le 7 juin dans le cas du Chevalier rouge $\left(18,5^{\circ} \mathrm{C}\right)$ alors qu'elles étaient toujours présentes à la fin de l'échantillonnage, le 23 juin, dans le cas du Chevalier de rivière, à une température de $19,0^{\circ} \mathrm{C}$.

Des femelles au stade VI apparaissent dans les captures le 17 mai pour le Chevalier blanc et le Chevalier rouge $\left(12,5^{\circ} \mathrm{C}\right)$ alors qu'elles sont présentes pour le Chevalier de rivière à compter du 10 juin $\left(18,5^{\circ} \mathrm{C}\right)$. Les dernières femelles de stade VI ont été capturées le 31 mai pour le Chevalier blanc $\left(17,0^{\circ} \mathrm{C}\right)$ et le 17 juin pour le Chevalier rouge $\left(19,0^{\circ} \mathrm{C}\right)$, alors qu'elles étaient toujours présentes à la dernière date d'échantillonnage pour le Chevalier de rivière $\left(19,0^{\circ} \mathrm{C}\right)$.

\section{Discussion}

Si l'on ajoute aux 16 espèces citées dans le Tableau 2 celles rapportées par Houde et Fournier (1992), il est probable qu'environ 20 espèces se reproduisent dans le rapide Farmer's. D'autres espèces capturées pourraient potentiellement utiliser le secteur échantillonné de la rivière Gatineau pour frayer (Scott et Crossman 1974; Bernatchez et Giroux 2000) : le Méné émeraude, le Naseux des rapides, et possiblement le Méné à nageoires rouges et le Raseux-de-terre gris. Toutefois, la période d'échantillonnage ne correspondait pas à la période de fraie pour certaines espèces (e.g. Méné émeraude) alors que trop peu de spécimens ont été capturés pour d'autres afin de déterminer l'état reproductif (e.g. Naseux des rapides).

La frayère multispécifique des rapides de la rivière Gatineau compte autant d'espèces que celle de la rivière aux Pins, à Boucherville (Massé et al. 1988). Les deux frayères ont toutefois des caractéristiques très différentes; la frayère de la rivière aux Pins constitue un habitat d'eau calme utilisé particulièrement par les espèces phytophyles. D'ailleurs, seulement trois espèces sont communes aux deux frayères : la Ouitouche, le Meunier noir et le Crapet de roche. Dans les eaux vives, Massé et al. (1988) mentionnent cinq autres frayères entre Carillon (rivière des Outaouais) et la rivière des Prairies qui comptent entre 8 et 27 espèces reproductrices. La rivière Gatineau se situe donc dans le milieu du peloton quant à son utilisation multispécifique.

Des femelles en état de fraie de Chevaliers rouge et blanc sont apparues sur le site en même temps le 10 mai. Elles y ont été capturées pendant une période de 10 jours dans le cas du Chevalier blanc et de 28 jours dans le cas du Chevalier rouge. La fraie des deux espèces a donc été en partie simultanée (Figures 3 et 4).

Contrairement à nos résultats, Curry et Spacie (1984) ont noté que le Chevalier blanc a amorcé la reproduction une semaine avant le Chevalier rouge dans le Deer Creek, Indiana, en 1979. Toutefois, Mongeau et al. $(1986,1992)$ ont également observé une simultanéité de la reproduction du Chevalier blanc et du Chevalier rouge dans leur étude sur la rivière Richelieu. En effet, ils ont observé que la reproduction de ces deux espèces débutait à la mi-mai pour se terminer à la mi-juin. Nos données semblent tendre vers le même résultat puisque des individus de stade VI ont été capturés du 17 mai au 21 juin pour les deux espèces (Figure 3 ). Mongeau et al. $(1986,1992)$ ont noté que le Chevalier rouge et le Chevalier blanc commençaient à déposer des œufs à une température de $10,7^{\circ} \mathrm{C}$. Meyer (1962) a indiqué que la température à laquelle la reproduction débutait pour le Chevalier rouge et le Chevalier blanc sur la rivière Des Moines, en Iowa, était $11,1^{\circ} \mathrm{C}$ et $13,3^{\circ} \mathrm{C}$ respectivement. Par contre, Burr et Morris (1977) ont observé une température de $16,0^{\circ} \mathrm{C}$ pour la reproduction du Chevalier rouge dans le ruisseau Big Rock, en Illinois et Hackney et al. (1970), une température de $14,4^{\circ} \mathrm{C}$ pour le Chevalier blanc dans la rivière Flint, en Alabama. La Figure 2 montre que la température de l'eau au 17 mai était à $12,5^{\circ} \mathrm{C}$, ce qui correspond relativement bien aux données de Meyer (1962) et de Mongeau et al. (1986, 1992).

Burr et Morris (1977) indiquent que plusieurs espèces de chevaliers pourraient possiblement frayer à des périodes et des températures similaires et sur des substrats comparables. Il peut toutefois y avoir de la ségrégation spatiale entre les espèces. En effet, le Chevalier rouge et le Chevalier doré (Moxostoma erythrurum) ont été observés en train de frayer simultanément au même site, mais le Chevalier doré avait une distribution plus étendue en amont, ce qui lui fournissait un habitat non utilisé par le Chevalier rouge (Curry et Spacie 1984). Par ailleurs, une étude comparant la fraie chez le Chevalier doré et le Chevalier noir (Moxostoma duquesnei) a démontré que ces deux espèces se reproduisaient au même moment et dans la même section du Stony Creek, en Illinois (Kwak et Skelly 1992). Les auteurs mentionnent toutefois qu'il existe des différences dans le choix des microhabitats de fraie et dans le comportement reproducteur et que celles-ci serviraient possiblement à réduire la compétition entre ces espèces. Bien qu'une étude plus approfondie de l'habitat et du comportement reproductif soit nécessaire, il est possible qu'une ségrégation spatiale et/ou comportementale se produise pour le Chevalier blanc et le Chevalier rouge dans la rivière Gatineau.

Des femelles de stade $\mathrm{V}$ du Chevalier de rivière ont été capturées pour la première fois le 3 juin et ce, jusqu'à la dernière journée d'échantillonnage. Sa re- 

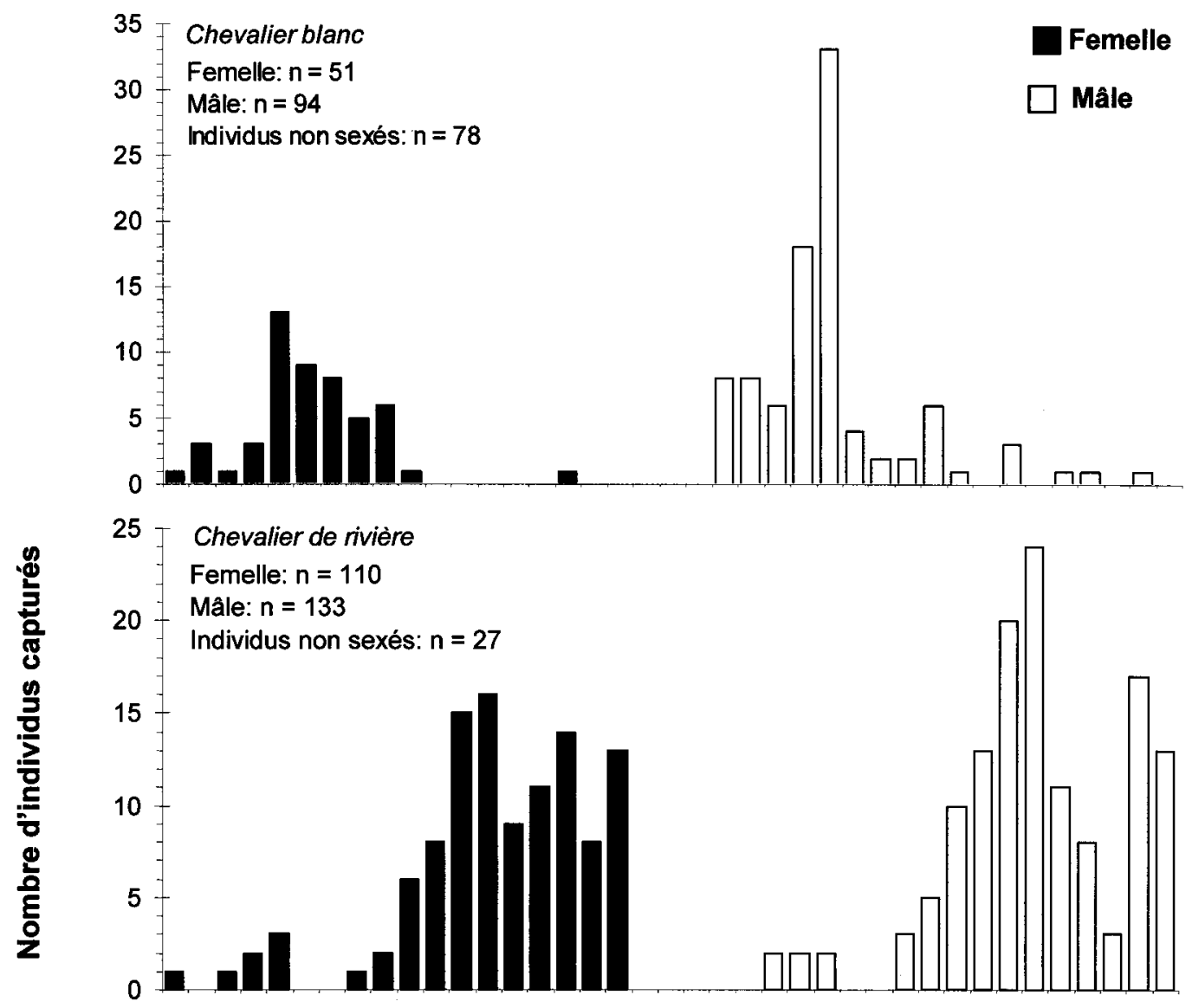

20 T Chevalier rouge

Femelle: $n=70$

Mâle: $n=108$

15 Individus non sexés: $\mathrm{n}=76$



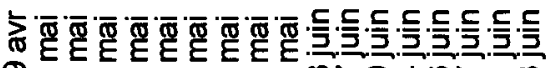
ำ



FIGURE 4. Nombre d'individus capturés par jour selon le sexe pour les trois espèces de chevalier, rivière Gatineau, printemps, 1999. 
production débute donc plus tard, soit autour du 10 juin (Figure 3) à une température de $18,5^{\circ} \mathrm{C}$. Mongeau et al. $(1986,1992)$ ont obtenu plus ou moins les mêmes résultats puisqu'ils indiquent que le Chevalier de rivière amorce la fraie autour de la deuxième semaine de juin à une température de $17,7^{\circ} \mathrm{C}$, une conclusion fondée sur la capture de deux spécimens au stade VI. Hackney et al. (1970) mentionnent également que le Chevalier de rivière a une reproduction tardive vis-à-vis de celle du Chevalier blanc dans la rivière Duck, au Tennessee. La différence entre la période de reproduction de cette espèce et celles des deux autres chevaliers présents dans les rapides de la rivière Gatineau est probablement due au fait que le Chevalier de rivière nécessite une température de l'eau plus élevée pour débuter sa reproduction.

Par ailleurs, pour ces trois espèces, nous observons des mâles de stade $\mathrm{V}$ à une date plus hâtive que les femelles au même stade (Figure 3). Ceci s'explique par le fait que les mâles du Chevalier blanc, du Chevalier rouge et du Chevalier de rivière précèdent les femelles sur les frayères (Meyer 1962; Hackney et al. 1968, 1970; Scott et Crossman 1974). Ce phénomène est d'ailleurs observé chez d'autres chevaliers et chez plusieurs autres Catostomidae (Meyer 1962; Scott et Crossman 1974; Jenkins et Jenkins 1980; Page et Johnston 1990; Kwak et Skelly 1992; Cooke et Bunt 1999).

Dans nos captures, le nombre de mâles de stade V est plus grand que celui de femelles pour les trois espèces de chevalier. Ceci n'est pas inhabituel. Hackney et al. (1970) ont observé un rapport des sexes de 4 mâles : 1 femelle sur une frayère de Chevalier blanc. De plus, Meyer (1962) note que chez les Chevaliers rouge et blanc, les femelles demeurent sur les sites de fraie beaucoup moins longtemps que les mâles. La fraie chez les chevaliers, et chez la plupart des Catostomidae, se fait généralement en présence de deux mâles et d'une femelle (Hackney et al. 1968; Burr et Morris 1977; Jenkins et Jenkins 1980; Page et Johnston 1990; Kwak et Skelly 1992; Cooke et Bunt 1999), d'où la nécessité d'une présence en plus grand nombre de mâles sur la frayère tel que nous l'avons observé pour chacune des trois espèces présentes au rapide Farmer's.

Nos travaux ont permis de découvrir le site de reproduction de l'une des plus importantes populations de Chevalier de rivière. Un échantillonnage sur quelques années serait nécessaire pour préciser la chronoséquence de fraie de cette espèce et des autres chevaliers utilisant le site. Un échantillonnage sur une plus longue période aurait probablement pu permettre de confirmer la fraie au rapide Farmer's d'un plus grand nombre d'espèces.

Cette étude démontre donc que le rapide Farmer's constitue une frayère multispécifique importante tant par le nombre d'espèces qui s'y reproduisent que par la présence d'espèces évaluées par le COSEPAC comme ayant une situation préoccupante (Lamproie du nord, Chevalier de rivière) ou même menacée (Fouille-roche gris). Localisés en plein coeur d'une zone urbaine, ces rapides ont une grande valeur écologique et doivent être protégés.

\section{Remerciements}

Nous remercions Anne Phelps, Annie-Chantal Guibord et Kevin Moon pour leur aide sur le terrain. Cette recherche a été partiellement subventionnée par une subvention à la découverte du Conseil de recherches en sciences naturelles et en genie du Canada (CRSNG) [François Chapleau].

\section{Littérature Citée}

Bernatchez, L., et M. Giroux. 2000. Les poissons d'eau douce du Québec et, leur répartition dans l'Est du Canada. Broquet, Boucherville, Québec. 350 pages.

Burr, B. M., et M. A. Morris. 1977. Spawning behavior of the Shorthead Redhorse, Moxostoma macrolepidotum, in Big Rock Creek, Illinois. Transactions of the American Fisheries Society 106: 80-82.

Cooke, S. J., et C. M. Bunt. 1999. Spawning and reproductive biology of the Greater Redhorse, Moxostoma valenciennesi, in the Grand River, Ontario. Canadian FieldNaturalist 113: 497-502.

COSEPAC. 2003. Résultats des évaluations du COSEPAC, novembre 2003. Comité sur la situation des espèces en péril au Canada, Ottawa, Ontario. 54 pages.

Curry, K. D., et A. Spacie. 1984. Differential use of stream habitat by spawning catostomids. American Midland Naturalist 111: 267-279.

Hackney, P.A., W.M. Tatum, et S.L. Spencer. 1968. Life history study of the River Redhorse, Moxostoma carinatum (Cope), in the Cahaba River, Alabama, with notes on the management of the species as a sport fish. Proceedings of the Annual Conference of the Southeastern Association of Game and Fish Commissioners 21: 324-332.

Hackney, P. A., G. R. Hooper, et J. F. Webb. 1970. Spawning behavior, age and growth, and sport fishery for the Silver Redhorse, Moxostoma anisurum (Rafinesque), in the Flint River, Alabama. Proceedings of the Twenty-Fourth Annual Conference, Southeastern Association of Game and Fish Commissioners 1970: 569-576.

Houde, P. et H. Fournier. 1992. Travaux de recherche de frayères dans la rivière des Outaouais et ses principaux affluents au cours du printemps 1989. Ministère du Loisir, de la Chasse et de la Pêche, Service de l'aménagement et de l'exploitation de la faune, Hull. 28 pages.

Jenkins, R. E., et D. J. Jenkins. 1980. Reproductive behavior of the Greater Redhorse, Moxostoma valenciennesi, in the Thousand Islands region. Canadian Field-Naturalist 94: 426-430.

Kwak, T. J., et T. M. Skelly. 1992. Spawning habitat, behavior, and morphology as isolating mechanisms of the Golden Redhorse, Moxostoma erythrurum, and the Black Redhorse, M. duquesnei, two syntopic fishes. Environmental Biology of Fishes 34: 127-137.

Massé, G., R. Fortin, P. Dumont, et J. Ferraris. 1988. Étude et aménagement de la frayère multispécifique de la rivière aux Pins et dynamique de la population de Grand brochet, Esox lucius L., du fleuve Saint-Laurent, Boucherville, Québec. Ministère du Loisir, de la Chasse et de la Pêche, Service de l'aménagement et de l'exploitation de la faune, Montréal, Québec, Rapport technique 06-40. 224 pages. 
Meyer, W. H. 1962. Life history of three species of redhorse (Moxostoma) in the Des Moines River, Iowa. Transactions of the American Fisheries Society 91: 412-419.

Mongeau, J.-R., P. Dumont, et L. Cloutier. 1986. La biologie du Suceur cuivré, Moxostoma hubbsi, une espèce rare et endémique à la région de Montréal, Québec, Canada. Ministère du Loisir, de la Chasse et de la Pêche, Service de l'aménagement et de l'exploitation de la faune, Montréal, Québec, Rapport technique 06-39. 137 pages.

Mongeau, J.-R., P. Dumont, et L. Cloutier. 1992. La biologie du Suceur cuivré (Moxostoma hubbsi) comparée à celle de quatre autres espèces de Moxostoma (M. anisurum, $M$. carinatum, M. macrolepidotum, M. valenciennesi). Revue canadienne de zoologie 70: 1354-1363.
Nikolsky, G. V. 1963. The ecology of fishes. Traduit par L. Birkett. Academic Press, New York. 352 pages.

Page, L. M., et C. E. Johnston. 1990. Spawning in the Creek Chubsucker, Erimyzon oblongus, with a review of spawning behavior in suckers (Catostomidae). Environmental Biology of Fishes 27: 265-272.

Scott, W. B., et E. J. Crossman. 1974. Poissons d'eau douce du Canada. Office des recherches sur les pêcheries du Canada Bulletin 184. 1026 pages.

Received 3 October 2003

Accepted 21 June 2004 